TRANSACTIONS OF THE

AMERICAN MATHEMATICAL SOCIETY

Volume 349, Number 10, October 1997, Pages 3875-3892

S 0002-9947(97)01945-4

\title{
INCOMPRESSIBLE REACTING FLOWS
}

\author{
JOEL D. AVRIN
}

\begin{abstract}
We establish steady-state convergence results for a system of reaction-convection-diffusion equations that model in particular combustion phenomena in the presence of nontrivial incompressible fluid motion. Despite the presence of the convection terms, we find that the asymptotic behavior of the system is identical to the case we have previously considered in which the velocity field was set equal to zero. In particular we are again able to establish the convergence of solutions to steady-states and to explicitly calculate the steady-states from the initial and boundary data. Key to our analysis is the establishment of high-order uniform bounds on the temperature and mass fraction components, a process significantly complicated by the presence of the convection terms.
\end{abstract}

\section{INTRODUCTION}

We consider reaction-convection-diffusion equations that model a general class of chemical kinetics phenomena that includes a variety of combustion problems. Unlike our previous studies of models of laminar flames (see e.g. [2], [3], [4], [5]), we consider models that allow for nontrivial fluid motion. In particular we focus here on cases where the fluid is incompressible.

We also focus on one-step reactions, although as in [4] we allow for multiple reactants. A simple example of such a reaction is

$$
2 \mathrm{NO}+\mathrm{Cl}_{2} \rightarrow 2 \mathrm{NOCl} .
$$

The general one-step reaction involving the reactants and products $A_{1}, \ldots, A_{N}$ can be written as

$$
\sum_{i=1}^{N} \nu_{i} A_{i} \rightarrow \sum_{i=1}^{N} \mu_{i} A_{i}
$$

where the stoichiometric coefficients $\nu_{i}$ and $\mu_{i}$ are positive integers. In (1.1), for example, if $A_{1}=N O, A_{2}=C l_{2}$, and $A_{3}=N O C l$, then $v_{1}=\mu_{3}=2, \nu_{2}=1$, and $\nu_{3}=\mu_{1}=\mu_{2}=0$.

Without loss of generality we can assume that $A_{1}, \ldots, A_{M}$ are the reactants for some $M$ with $1 \leq M<N$; i.e. $\nu_{i} \neq 0$ if and only if $1 \leq i \leq M$. Let $Y_{i}=Y_{i}(x, t)$ denote the respective mass fractions of the $A_{i}, i=1, \ldots, M$; let $T=T(x, t)$ denote the (dimensionless) temperature; and let $v=\left(v_{1}, \ldots, v_{n}\right), v_{i}=v_{i}(x, t)$, denote the velocity of the fluid. Here $x \in \Omega$, a bounded domain in $\mathbb{R}^{n}$ with smooth boundary $\partial \Omega$, and $t \geq 0$.

Received by the editors September 20, 1994.

1991 Mathematics Subject Classification. Primary 35B40, 35K55, 35K57, 35Q10, 80A25.

(C) 1997 American Mathematical Society 
Let $f(T)$ denote the rate law of the reaction, e.g. for positive constants $B$ and $E$ $f(T)=B \exp (-E / T)$ in typical combustion situations (see e.g. [8], [30]). In other chemical kinetics situations the temperature dependence can be neglected and the reaction rate is taken to be a constant, i.e. $f(T) \equiv B$ (see e.g. [9]). We take $f$ to be any smooth function satisfying the following assumptions:

(i) $f(T) \geq 0 \forall T \in \mathbb{R}$,

(ii) $f$ and all its derivatives are uniformly bounded in $T$,

(iii) $f$ is monotonically increasing in $T$.

Note that assumptions (i)-(iii) are in particular satisfied by the examples of $f$ given above. For $\nu_{i}$ as above and for $f$ satisfying (i)-(iii) we set

$$
\omega=\left[\prod_{i=1}^{M} Y_{i}^{\nu_{i}}\right] f(T) .
$$

Set $\alpha_{i}=m_{i} \nu_{i}, 1 \leq i \leq M$, where $m_{i}$ is the molecular mass of the $i$ th species. Let $Q, d_{1}, d_{2}, \ldots, d_{M}$ be positive constants, where $Q$ denotes the heat release of the reaction (1.2). Then the following system of reaction-convection-diffusion equations models an incompressible reacting flow wherein the reaction proceeds according to the scheme (1.2):

$$
\begin{gathered}
T_{t}=\Delta T-v \cdot \nabla T+Q w \\
Y_{i t}=d_{i} Y_{i}-v \cdot \nabla Y_{i}-\alpha_{i} \omega, \quad i=1, \ldots, M .
\end{gathered}
$$

We will show below how (1.4) can be derived from the equations describing general reacting flows.

In what follows we will consider (1.4) together with one of the following sets of boundary conditions on $T$ and $Y_{i}$ : we will assume that either

$$
\left.\frac{\partial T}{\partial \nu}\right|_{\partial \Omega}=\left.\frac{\partial Y_{i}}{\partial \nu}\right|_{\partial \Omega}=0, \quad 1 \leq i \leq M
$$

where $\nu$ is the outward normal on $\Omega$, or, for a sufficiently smooth function $g$ on $\partial \Omega$, that

$$
\begin{gathered}
\left.T\right|_{\partial \Omega}=g, \\
\left.\frac{\partial Y_{i}}{\partial \nu}\right|_{\partial \Omega}=0, \quad 1 \leq i \leq M .
\end{gathered}
$$

Meanwhile $v$ satisfies the incompressible Navier-Stokes system

$$
\begin{gathered}
v_{t}=\mu \Delta v-(v \cdot \nabla) v-\nabla p, \\
\operatorname{div} v=0
\end{gathered}
$$

in $\Omega$, where the positive constant $\mu$ is the reciprocal of the Reynolds number, and $p=p(x, t)$ denotes the fluid pressure. For $v$ we assume the standard "no-slip" boundary condition

$$
\left.v\right|_{\partial \Omega}=0 .
$$

For further physical background on (1.7), (1.8) there are, of course, many references; the discussion in e.g. [17], [25], [26] is appropriate to our purposes here.

The equations (1.4)-(1.8) represent a special case of systems of equations describing more general reactive flows. We do not intend to reproduce the physical 
derivation here; instead we list below the general system as described in e.g. [8], [30] and refer the reader to [8], [30] for discussions of the physical background:

$$
\begin{gathered}
\rho_{t}+\nabla \cdot(\rho v)=0, \\
\rho\left(v_{t}+(v \cdot \nabla) v\right)=-\nabla p+\mu\left[\Delta v+\frac{1}{3} \nabla(\nabla \cdot v)\right], \\
\rho\left(T_{t}+v \cdot \nabla T\right)=\Delta T+Q \omega, \\
\rho\left(Y_{i t}+v \cdot \nabla Y_{i}\right)=d_{i} \Delta Y_{i}-\alpha_{i} \omega, \quad 1 \leq i \leq M .
\end{gathered}
$$

Here $\rho=\rho(x, t)$ is the density of the fluid. When the fluid is incompressible, then the density is constant (see e.g. [25]); without loss of generality we take $\rho \equiv 1$. Taking this assumption together with the observation that the term $(1 / 3) \nabla(\nabla \cdot v)$ can be removed via absorption into the term $\Delta v$ by a rotation transformation, we thus obtain the equations (1.4), (1.7). Regimes in which the constant-density assumption is valid are discussed e.g. in [8, pp. 25-26]. In particular, the assumption makes sense if the heat release is small, which occurs, for example, in dilute mixtures, a situation assumed throughout [8], [30].

There is a wide body of literature devoted to obtaining rigorous results on the qualitative behavior of (1.4) with $v=0$, in which (1.4) reduces to a reactiondiffusion system. The basic reaction $A \rightarrow B$ (i.e. $M=1$ ) has been studied in this case, both when $\Omega=\mathbb{R}$ and on bounded domains (see e.g. [2], [7], [11], [13], [19], [20], [22], [23], [27], [29] and the references contained therein). The case $v=0$ and $M$ arbitrary was studied in [4], wherein the convergence of solutions to steadystates was established, and the steady-states were explicitly calculated from the initial data in the case of conditions (1.5) and from the initial and boundary data in the case of conditions (1.6). The purpose of the present work will be to show in the case $n \leq 3$ that the same steady-state-convergence results hold in the presence of nontrivial incompressible fluid motion if $v$ in (1.7) is smooth enough.

Before stating our main result, we note the following theorem, which establishes the basic existence, uniqueness, and regularity properties of our solutions to (1.4), given a smooth solution $v$ of (1.7). Here we set $T_{0}(x)=T(x, 0)$ and $Y_{i 0}(x)=$ $Y_{i}(x, 0)$.

Theorem 1.1. Let $T_{0}$ and $Y_{i 0}, 1 \leq i \leq m$, be non-negative functions in $C(\bar{\Omega})$. Given integers $k \geq 2$ and $j \geq 1$, suppose that $v$ is a strong solution of (1.7) satisfying $v \in C_{B}^{j}\left((\eta,+\infty) ; C^{k}(\bar{\Omega})\right)$ for each $\eta>0$, and suppose that $g \in C(\partial \Omega)$ is non-negative and smooth enough so that the solution $w$ of the Dirichlet problem

$$
\begin{gathered}
\Delta w=0 \text { in } \Omega \\
\left.w\right|_{\partial \Omega}=g
\end{gathered}
$$

satisfies $w \in C^{k}(\bar{\Omega})$. Then there exist unique non-negative global strong solutions $T, Y_{i}, 1 \leq i \leq m$, of (1.4) satisfying $T, Y_{i} \in C([0,+\infty) ; C(\bar{\Omega})) \cap C^{j}((0,+\infty)$; $\left.C^{k}(\bar{\Omega})\right)$.

This result is a fairly straightforward generalization of [4, Theorem 1.1], and the proof is fairly standard and can safely be omitted. The main point is that the techniques of [24, Chap. 14] still apply when the convection terms are added.

Now we set, for any $h \in C(\bar{\Omega})$,

$$
h_{A V}=\frac{1}{|\Omega|} \int_{\Omega} h(x) d x .
$$


Let $\left(Y_{i 0}\right)_{A V}$ be the right-hand side of (1.11) with $h$ replaced by $Y_{i 0}, 1 \leq i \leq$ $m$. As in [4], we can, by relabeling if necessary, assume that $\left(1 / \alpha_{1}\right)\left(Y_{10}\right)_{A V}=$ $\min _{1 \leq i \leq M}\left\{\left(1 / \alpha_{i}\right)\left(Y_{i 0}\right)_{A V}\right\}$. Set $\left(Z_{i 0}\right)=\left(1 / \alpha_{i}\right)\left(Y_{i 0}\right)_{A V}-\left(1 / \alpha_{1}\right)\left(Y_{10}\right)_{A V}, 1 \leq i \leq$ $M$; then each $\left(Z_{i 0}\right)_{A V}$ is non-negative. Finally, set

$$
\left(G_{0}\right)_{A V}=\left(T_{0}\right)_{A V}+\left(Q / \alpha_{1}\right)\left(Y_{10}\right)_{A V} .
$$

We are now ready to state our main result.

Theorem 1.2. Let $n \leq 3$; let $T_{0}, Y_{i 0}$, and $Z_{i 0}, 1 \leq i \leq M$, be as above; and let $v$ and $g$ satisfy the conditions of Theorem 1.1. Suppose additionally that $T_{0}$ is not identically zero and that $g(x)>0$ for all $x \in \partial \Omega$. Then

$$
\lim _{t \rightarrow \infty} Y_{i}(x, t)=\alpha_{i}\left(Z_{i 0}\right)_{A V}, \quad 1 \leq i \leq M,
$$

uniformly in $x$. If conditions (1.5) are assumed, then

$$
\lim _{t \rightarrow \infty} T(x, t)=\left(G_{0}\right)_{A V}
$$

uniformly in $x$, while if conditions (1.6) are assumed,

$$
\lim _{t \rightarrow \infty} T(x, t)=w(x)
$$

uniformly in $x$, where $w$ is as in (1.10).

Remarks. (a) Note that when $i=1,(1.12)$ asserts that $Y_{1}$ converges uniformly to zero. As in [4] we will actually establish this first in the proof and then obtain (1.12) for $2 \leq i \leq M$.

(b) As previously mentioned, the steady-state-convergence results contained in Theorem (1.2) are identical to the $v=0$ case handled in [4]. The physical intuition is that, whatever enhancing or inhibiting effects to the mixing are present due to the convection, the diffusion of the species and temperature take over in the long term to allow the reaction to fully complete itself; this is evidently the case, at least, when the velocity field is smooth. We will discuss below under what circumstances smooth strong solutions of (1.7) are known to exist. At any rate, as in [4] we see that a residual amount of $A_{i}$ always remains unless the initial amount of $A_{i}$ versus the initial amount of $A_{1}$ is carefully balanced, i.e. $\left(Z_{i 0}\right)_{A V}=0$.

(c) We have restricted ourselves to spatial domains of dimension $n \leq 3$ for technical reasons; we will see at a certain juncture below that this assumption arises due to a particular use of the Sobolev inequalities. While we do not see immediately how to work around this technical detail, we have a fair amount of confidence that in fact it can be done and that the restriction on $n$ can thus be removed.

(d) The assumption that $T_{0}$ be not identically zero is entirely reasonable, since otherwise no reaction would occur. Meanwhile, the assumption $g>0$ is somewhat more restrictive and is assumed here for mainly technical reasons. There is certainly a precedent, however, for a positivity assumption on $g$ : see e.g. [13], [23] and [17, $\S 5.1,6.1$, and 10.2] wherein the condition $g \equiv 1$ is assumed in a reaction of the form $A \rightarrow B$.

We will prove Theorem 1.2 in Section 3 below. In Section 2 we will discuss some preliminaries and some technical results. We now discuss under what circumstances solutions $v$ of the incompressible Navier-Stokes equations (1.7) possess the desired properties needed in Theorems 1.1 and 1.2. 
The global existence and uniqueness of strong solutions of (1.7) have been known for many years when $n=2$ (see e.g. [17], [26]). For this case global bounds on all derivatives for $t>0$ that guarantee the conditions on $v$ needed in Theorems 1.1 and 1.2 were established in [15]; this result was recovered with a different proof in [6, section 3]. When $n=3$ it is well known that the situation is quite different; the existence and uniqueness of global strong solutions remains an open question for arbitrary initial data. Indeed, for many years global existence was known only for small initial data in the domain of $(-\Delta)^{1 / 4}[12]$; this result was generalized to allow small initial data in $L^{3}(\Omega)$ in [14]. Recently, it was discovered that large initial data could be allowed if the domain $\Omega$ is "thin" enough. The first result of this type appeared in [21], in which domains of the form $\Omega=\Omega^{\prime} \times(0, \varepsilon)$ were considered, where $\Omega^{\prime}$ is a rectangle in $\mathbb{R}^{2}$ in the case of periodic boundary conditions, and $\Omega^{\prime}$ is an arbitrary domain in $\mathbb{R}^{2}$ in the case of mixed periodic-Dirichlet boundary conditions. The case of purely homogeneous Dirichlet boundary conditions (1.8) was handled in [6]; moreover we observed that on domains of the form $\Omega=\Omega^{\prime} \times(0, \varepsilon)$ the first eigenvalue $\lambda_{1}$ of $-\Delta$ under the conditions (1.8) is $O\left(\frac{1}{\varepsilon^{2}}\right)$. Motivated by this, we then went on to define a domain $\Omega$ to be thin in $\mathbb{R}^{3}$ if under the conditions (1.8) $\lambda_{1}$ is large; thus we were able to consider a more general class of "thin" domains in [6]. Meanwhile, global bounds on the derivatives of $v$ for $t>0$ were also established in both [6] and [21].

To provide a definite statement of a general class of circumstances when $n=3$ under which we have the existence of $v$ satisfying (1.7), (1.8), and the requisite conditions of Theorems 1.1 and 1.2, we restate and condense [6, Theorems 1.2, 1.3, an 1.4] below. For simplicity, we set the forcing term equal to zero, as we do above in (1.7).

Theorem 1.3. Let $X_{4}$ denote the closure in $L^{4}(\Omega)$ of $\left\{w \in C_{0}^{\infty}(\Omega) \mid \operatorname{div} w=0\right\}$, let $v_{0} \in X_{4}$, and let $\lambda_{1}$ be the first eigenvalue of $-\Delta$ under the conditions (1.8). Let $M=\left\|v_{0}\right\|_{4}$; then if $\left(1 / \lambda_{1}\right)^{1 / 8} M$ is small enough, there exists a unique global strong solution of (1.7) such that, for each $\alpha \geq 1$, there exists for each $\eta>0$ a constant $M_{\alpha}^{\eta}$ such that $\|v(t)\|_{\alpha, 2} \leq M_{\alpha}^{\eta}$ for all $t \geq \eta$.

This theorem is proven in [6] via a contraction mapping principle, together with a related argument to establish regularity for $t \geq \eta$. Hence " $\left(1 / \lambda_{1}\right)^{1 / 8} M$ is small enough" means that for an appropriate constant $K$ we have that $K\left(1 / \lambda_{1}\right)^{1 / 8} M<1$. The constant $K$ depends basically upon the constants appearing in various Sobolev inequalities and on various bounds concerning the Stokes semigroup $\exp (t P \Delta)$, where $P: L^{2}(\Omega) \rightarrow H_{\sigma}$ and $H_{\sigma}$ is the completion in $L^{2}(\Omega)$ of $\left\{w \in C_{0}^{\infty}(\Omega) \mid \operatorname{div} w=\right.$ $0\}$. Thus, loosely speaking, Theorem 1.3 allows $\left\|v_{0}\right\|_{4}$ to be $O\left(\lambda_{1}^{-1 / 8}\right)$.

Before turning to the developments in the next sections, we note that, while we allow for multiple species in our reaction mechanism, we have restricted ourselves for simplicity to one-step reactions. Meanwhile, there are a number of rigorous results available now on reaction schemes modeling multi-step reactions (see e.g. [3], $[5],[10],[28]$ and the references contained therein) in the constant-density approximation with $v=0$. We are confident that the techniques developed here could be applied in these complex chemistry cases to allow for nonzero $v$; we have avoided doing so here since otherwise we would have to develop separate a priori estimates (similar to the ones treated in the next section for (1.4)) for each of the equations corresponding to the different reaction schemes. We will, however, make general comments on how to accomplish these tasks in our concluding remarks. Another 
reason for mentioning the works [5], [10] in particular is that in them [10, Lemma $2.5]$ and extensions thereof played a central role; this was also the case in [4], and it will be the case here as well.

\section{Preliminary observations and Results}

We begin by showing the existence of some conserved quantities. Such quantities also play a large role in [4]; extending then to the case of nonzero $v$ depends on applying the following result:

Lemma 2.1. Let $v$ be as above; then for each $h \in H^{1}(\Omega)$ we have that

$$
\int_{\Omega} v \cdot \nabla h d x=0 .
$$

Proof. As above, let $H_{\sigma}$ be the completion in $L^{2}(\Omega)$ of $\left\{w \in C_{0}^{\infty}(\Omega) \mid \operatorname{div} w=\right.$ $0\}$. Then it is well known (see e.g. [12], [14]) that $\nabla h \in H_{\sigma}^{\perp}$, from which (2.1) immediately follows. One can also deduce this directly by noting the identity $v \cdot \nabla h=\operatorname{div}(h v)-(\operatorname{div} v) h=\operatorname{div}(h v)$ and then using Green's Theorem.

With this in mind, we set $Z_{i}(x, t)=\left(1 / \alpha_{i}\right) Y_{i}(x, t)-\left(1 / \alpha_{1}\right) Y_{1}(x, t), 1 \leq i \leq M$. Then by taking appropriate linear combinations in (1.4b) and integrating over $\Omega$, we have from Lemma 2.1 that

$$
\begin{aligned}
\frac{d}{d t} \int_{\Omega} Z_{i}(x, t) d x & =-\int_{\Omega} v \cdot \nabla\left(\alpha_{i}^{-1} Y_{i}(x, t)-\alpha_{1}^{-1} Y_{1}(x, t)\right) d x \\
& =0, \quad 1 \leq i \leq M
\end{aligned}
$$

If we set $\left(Z_{i}\right)_{A V}(t)$ equal to the right-hand side of (1.11) with $h$ replaced by $Z_{i}$, we can restate $(2.2)$ as

$$
\left(Z_{i}\right)_{A V}(t)=\left(Z_{i 0}\right)_{A V}, 1 \leq i \leq M
$$

for all $t \geq 0$, where $Z_{i 0}$ is as in (1.12).

Similarly, if conditions (1.5) are assumed, we set $G(x, t)=T(x, t)+\left(Q / \alpha_{1}\right) Y_{1}(x, t)$; then we have that

$$
\frac{d}{d t} \int_{\Omega} G(x, t) d x=-\int_{\Omega} v \cdot \nabla\left(T(x, t)+\left(Q / \alpha_{1}\right) Y_{1}(x, t)\right) d x=0
$$

i.e. that

$$
(G)_{A V}(t)=\left(G_{0}\right)_{A V}
$$

for all $t \geq 0$, where $\left(G_{0}\right)_{A V}$ is as in (1.13).

Equation (2.5) will allow us to develop a priori estimates for $T$ under the conditions (1.5). It is also already evident that spatial averages play a central role in determining further qualitative behavior of the solutions to (1.4). As in [4], [5], [10] the key idea that will connect (2.3) and (2.5) to the qualitative behavior of solutions will be to show that the $Y_{i}$ (and $T$ under the conditions (1.5)) behave for large time like their spatial averages (see Lemma 2.3 below). To do this we first need some strong a priori estimates. The situation is complicated by the convection terms in that, unlike the case $v=0$ considered in [4], [5], [10], the Schauder estimates do not directly apply.

Proposition 2.1. Let $T$ and $Y_{i}, 1 \leq i \leq M$, be the solutions to (1.4) given by Theorem 1.1. Then for all $t \geq 0$ we have that

$$
\left\|Y_{i}(\cdot, t)\right\|_{\infty} \leq\left\|Y_{i 0}\right\|_{\infty}, \quad 1 \leq i \leq M,
$$


and that for each $\eta>0$ and each integer $\alpha \geq 0$ there exist constants $K_{\alpha, \infty}^{\eta}$ and $L_{\alpha, \infty}^{\eta}$ such that

$$
\left\|D^{\alpha} T(\cdot, t)\right\|_{\infty} \leq K_{\alpha, \infty}^{\eta}
$$

and

$$
\left\|D^{\alpha} Y_{i}\right\|_{\infty} \leq L_{\alpha, \infty}^{\eta}
$$

for all $t \geq \eta$.

Proof. We first note that (2.6) follows immediately from the positivity of the $Y_{i}$ and of $f(T)$ and the maximum principle (see e.g. [24]). For (2.7) and (2.8), we employ a bootstrap process. Let $Q_{t}=\Omega \times[0, t]$ for each $t>0$. Integrating (1.4b) over $Q_{t}$, we obtain

$$
\left\|Y_{i}(\cdot, t)\right\|_{1}+\alpha_{i} \int_{0}^{t} \int_{\Omega} \omega(x, s) d x d s=\left\|Y_{i 0}\right\|_{1}, \quad 1 \leq i \leq M,
$$

where we have used the positivity of the $Y_{i}$ and (2.1) with $h=Y_{i}$. Thus, in particular,

$$
\int_{0}^{\infty} \int_{\Omega} \omega(x, s) d x d s \leq\left(1 / \alpha_{1}\right)\left\|Y_{10}\right\|_{1} .
$$

Let $\left(\right.$, ) denote the inner product on $L^{2}(\Omega)$; then for any $h_{1}, h_{2} \in H^{1}(\Omega)$ we have, integrating by parts and using $(1.7 \mathrm{~b})$ and $(1.8)$, that $\left(v \cdot \nabla h_{1}, h_{2}\right)=-\left(h_{1}, v \cdot \nabla h_{2}\right)$. Setting $h_{1}=h_{2}=h$, we see that

$$
(v \cdot \nabla h, h)=0
$$

for all $h \in H^{1}(\Omega)$. Thus if we multiply (1.4b) by $Y_{i}$ and integrate over $Q_{t}$, we obtain

$$
\frac{1}{2}\left\|Y_{i}(\cdot, t)\right\|_{2}^{2}+d_{i} \int_{0}^{t}\left\|\nabla Y_{i}\right\|_{2}^{2} d s \leq \frac{1}{2}\left\|Y_{i 0}\right\|_{2}^{2}, \quad 1 \leq i \leq M,
$$

where we have used the fact that $-\alpha_{i} \omega Y_{i}$ is negative for all $(x, t) \in Q_{t}$.

Now we multiply both sides of (1.4a) and integrate over $\Omega$ to obtain that

$$
\begin{aligned}
& \frac{1}{2} \frac{d}{d t}\|T(\cdot, t)\|_{2}^{2}=-\|\nabla T\|_{2}^{2}+Q(\omega, T) \\
& \leq-\|\nabla T\|_{2}^{2}+\frac{Q}{\varepsilon}\|\omega\|_{2}^{2}+Q \varepsilon\|T\|_{2}^{2} \\
& \leq-\|\nabla T\|_{2}^{2}+\frac{Q}{\varepsilon}\|\omega\|_{\infty}\|\omega\|_{1}+Q \varepsilon\|T\|_{2}^{2} \\
& \leq-\|\nabla T\|_{2}^{2}+\frac{Q B}{\varepsilon}\left[\prod_{i=1}^{M}\left\|Y_{i 0}\right\|_{\infty}\right]\|\omega\|_{1}+Q \varepsilon\|T\|_{2}^{2} \\
& \equiv-\|\nabla T\|_{2}^{2}+(Q / \varepsilon) C_{\omega}\|\omega\|_{1}+Q \varepsilon\|T\|_{2}^{2}
\end{aligned}
$$

where $\varepsilon>0$ will be chosen below; here we have used (2.6) and set $B=\|f\|_{\infty}$, so that $\|\omega\|_{\infty} \leq C_{\omega}$.

We complete the estimate (2.13) differently, depending on which boundary conditions are used. Under conditions (1.6) we use the Poincaré inequality

$$
\|T\|_{2}^{2} \leq\left(1 / \lambda_{1}\right)\|\nabla T\|_{2}^{2}
$$


and set $\varepsilon=\lambda_{1} / 2 Q$, so that from (2.13) we obtain

$$
\frac{1}{2} \frac{d}{d t}\|T(\cdot, t)\|_{2}^{2} \leq-\frac{1}{2}\|\nabla T\|_{2}^{2}+\left(2 Q^{2} / \lambda_{1}\right) C_{\omega}\|w\|_{1} .
$$

Integrating (2.15) over $[0, T]$ and using (2.10), we obtain

$$
\|T(\cdot, t)\|_{2}^{2}+\int_{0}^{t}\|\nabla T\|_{2}^{2} d s \leq\left(4 Q^{2} /\left(\alpha_{1} \lambda_{1}\right)\right) C_{\omega}\left\|Y_{10}\right\|_{1}+\left\|T_{0}\right\|_{2}^{2} .
$$

Under conditions (1.5) we set $T_{1}=T-(T)_{A V}$, and let $\gamma_{1}$ be the first nonzero eigenvalue of $-\Delta$; then e.g. by eigenfunction expansion we see that

$$
\left\|T_{1}\right\|_{2}^{2} \leq\left(1 / \gamma_{1}\right)\left\|\nabla T_{1}\right\|_{2}^{2}=\left(1 / \gamma_{1}\right)\|\nabla T\|_{2}^{2} .
$$

Thus, noting that (2.5) and the non-negativity of $Y_{1}$ imply that $(T)_{A V} \leq\left(G_{0}\right)_{A V}$, we have that

$$
\begin{aligned}
(\omega, T) & \leq\left(G_{0}\right)_{A V}\|\omega\|_{1}+\left(\omega, T_{1}\right) \\
& \leq\left(G_{0}\right)_{A V}\|\omega\|_{1}+\frac{1}{\varepsilon}\|\omega\|_{2}^{2}+\varepsilon\left\|T_{1}\right\|_{2}^{2} \\
& \leq\left[\left(G_{0}\right)_{A V}+\left(\frac{1}{\varepsilon}\right) C_{\omega}\right]\|\omega\|_{1}+\left(\varepsilon / \gamma_{1}\right)\|\nabla T\|_{2}^{2} .
\end{aligned}
$$

Setting $\varepsilon=\gamma_{1} / 2 Q$ and using the first line of (2.13), we have that

$$
\frac{1}{2} \frac{d}{d t}\|T(\cdot, t)\|_{2}^{2} \leq-\frac{1}{2}\|\nabla T\|_{2}^{2}+Q\left[\left(G_{0}\right)_{A V}+\left(\frac{2 Q}{\gamma_{1}}\right) C_{\omega}\right]\|\omega\|_{1} .
$$

Integrating (2.19) over $[0, t]$ and using (2.10), we obtain

$$
\|T(\cdot, t)\|_{2}^{2}+\int_{0}^{t}\|\nabla T\|_{2}^{2} d s \leq \frac{2 Q}{\alpha_{1}}\left[\left(G_{0}\right)_{A V}+\left(\frac{2 Q}{\gamma_{1}}\right) C_{\omega}\right]\left\|Y_{10}\right\|_{1}+\left\|T_{0}\right\|_{2}^{2} .
$$

Thus from (2.16) and (2.20) we see that under either of the conditions (1.5) and (1.6) there exists a constant $C_{0}^{T}$ such that

$$
\|T(\cdot, t)\|_{2}^{2}+\int_{0}^{t}\|\nabla T\|_{2}^{2} d s \leq C_{0}^{T}
$$

for all $t \geq 0$.

For the next step in the bootstrap process we multiply both sides of (1.4a) by $-\Delta T$, integrate over $\Omega$, on the left-hand side integrate by parts, and use the Cauchy-Schwartz inequality to obtain that

$$
\begin{aligned}
\frac{d}{d t} \frac{1}{2}\|\nabla T\|_{2}^{2}= & -\|\Delta T\|_{2}^{2}+(v \cdot \nabla T, \Delta T)+Q(\omega, \Delta T) \\
\leq & -\|\Delta T\|_{2}^{2}+\frac{1}{\varepsilon}\|v \cdot \nabla T\|_{2}^{2}+\varepsilon\|\Delta T\|_{2}^{2} \\
& +\frac{Q}{\varepsilon}\|\omega\|_{2}^{2}+Q \varepsilon\|\Delta T\|_{2}^{2} .
\end{aligned}
$$

Setting $\varepsilon=[2(1+Q)]^{-1}$, we see from (2.22) that

$$
\begin{aligned}
\frac{d}{d t} \frac{1}{2}\|\nabla T\|_{2}^{2} \leq & -\frac{1}{2}\|\Delta T\|_{2}^{2}+2(1+Q)\|v \cdot \nabla T\|_{2}^{2} \\
& +2 Q(1+Q)\|\omega\|_{2}^{2} .
\end{aligned}
$$

From this point on we will need to use the (unlimited) regularity of our solutions to $(1.4)$ and $(1.7)$ on intervals $[\eta,+\infty)$ for each $\eta>0$. By replacing our initial 
data $T_{0}, Y_{i 0}, v_{0}$ with $T(x, \eta), Y_{i}(x, \eta), v(x, \eta)$ for a fixed $\eta>0$ we can, without loss of generality, assume we have such regularity on $[0,+\infty)$. In particular, we can assume that for all $t \geq 0\|v(\cdot, t)\|_{\alpha, 2}$ is uniformly bounded for each $\alpha>0$. Thus from the Sobolev inequalities it follows that for each $\alpha>0$ there exists a constant $V_{\alpha}$ such that

$$
\left\|D^{\alpha} v(\cdot, t)\right\|_{\infty} \leq V_{\alpha}
$$

for all $t \geq 0$. Using (2.24) with $\alpha=0$, we thus have from (2.23) that

$$
\begin{aligned}
\frac{d}{d t}\|\nabla T\|_{2}^{2} & \leq-\|\Delta T\|_{2}^{2}+4(1+Q) V_{\alpha}^{2}\|\nabla T\|_{2}^{2}+4 Q(1+Q)\|w\|_{2}^{2} \\
& \leq-\|\Delta T\|_{2}^{2}+4(1+Q) V_{0}^{2}\|\nabla T\|_{2}^{2}+4 Q(1+Q) C_{\omega}\left\|\omega_{1}\right\|_{1}
\end{aligned}
$$

where $C_{\omega}$ is as in (2.13).

Integrating (2.25) on $[0, t]$ and using (2.10) and (2.21), we have for all $t \geq 0$ that

$$
\begin{aligned}
& \|\nabla T(\cdot, t)\|_{2}^{2}+\int_{0}^{t}\|\Delta T\|_{2}^{2} d s \leq 4(1+Q) V_{0}^{2} \int_{0}^{t}\|\nabla T\|_{2}^{2} d s \\
& \quad+4 Q(1+Q) C_{\omega} \int_{0}^{t}\|\omega\|_{1} d s \\
& \leq 4(1+Q) V_{0}^{2} C_{0}^{T}+4 Q(1+Q) C_{\omega}\left(1 / \alpha_{1}\right)\left\|Y_{10}\right\|_{1} \\
& \equiv C_{1}^{T} .
\end{aligned}
$$

By using arguments similar to those in (2.22)-(2.26) and using (2.12) instead of $(2.21)$, it follows that there exists a constant $C_{1}^{i}$ such that

$$
\left\|\nabla Y_{i}(\cdot, t)\right\|_{2}^{2}+d_{i} \int_{0}^{t}\left\|\Delta Y_{i}\right\|_{2}^{2} d s \leq C_{1}^{i}, \quad 1 \leq i \leq M,
$$

for all $t \geq 0$.

Before obtaining estimates on $\left\|D^{\alpha} T\right\|_{2}$ and $\left\|D^{\alpha} Y_{i}\right\|_{2}$ for $\alpha>1$ we need to calculate higher-order estimates on $\omega$ in terms of these quantities. We begin this process with an estimate based on a high-order version of the chain rule.

Lemma 2.2. Suppose that $g:[-U, U] \rightarrow \mathbb{R}$ is a smooth function whose derivatives are all bounded on $[-U, U]$, and suppose that $u: \Omega \rightarrow[-U, U]$ is a smooth function. Then for each integer $\alpha \geq 1$ there exists a constant $G_{\alpha}$ such that

$$
\left\|D^{\alpha}(g(u))\right\|_{2} \leq G_{\alpha}\left(\left\|D^{\alpha} u\right\|_{2}^{2}+\cdots+\left\|D^{\alpha} u\right\|_{2}^{2}\right)^{\alpha / 2} .
$$

Proof. The case $\alpha=1$ is bounded by the ordinary chain rule and the boundedness of $g^{\prime}$. Meanwhile, a higher-order version of the chain rule (see e.g. [16, p. 33]) states that

$$
D^{\alpha}(g(u))=\sum_{\beta} \frac{\alpha !}{i ! j ! h ! \ldots k !}\left[\left(D^{\beta} g\right)(u)\right]\left(\frac{D u}{1 !}\right)^{i}\left(\frac{D^{2} u}{2 !}\right)^{j}\left(\frac{D^{3} u}{3 !}\right)^{h} \cdots\left(\frac{D^{(l)} u}{l !}\right)^{k}
$$

where $i+2 j+3 h+\cdots+l k=\alpha$ and $\beta=i+j+h+\cdots+k$. Thus by the boundedness of $\left(D^{\beta} g\right)(u)$ we have that there exists a constant $G_{\alpha}^{\prime}$ such that

$$
\left\|D^{\alpha}(g(u))\right\|_{2} \leq G_{\alpha}^{\prime} \sum_{\beta}\left\|(D u)^{i}\left(D^{2} u\right)^{j}\left(D^{3} u\right)^{k} \cdots\left(D^{(l)} u\right)^{k}\right\|_{2} .
$$


But since $i / \alpha+(2 j) / \alpha+(3 h) / \alpha+\cdots+(l k) / \alpha=1$, we have by Hölder's inequality that

$$
\begin{aligned}
& \left\|(D u)^{i}\left(D^{2} u\right)^{j}\left(D^{3} u\right)^{h} \cdots\left(D^{(l)} u\right)^{k}\right\|_{2} \\
& \quad \leq\|D u\|_{2 \alpha / 1}^{i}\left\|D^{2} u\right\|_{2 \alpha / 2}^{j}\left\|D^{3} u\right\|_{2 \alpha / 3}^{h} \cdots\left\|D^{(l)} u\right\|_{2 \alpha / l}^{k} .
\end{aligned}
$$

Now for each $s=1,2, \ldots, l$ there exists by the Sobolev inequalities a constant $C_{s}$ such that (for $n=3$ )

$$
\left\|D^{s} u\right\|_{2 \alpha / s} \leq C_{s}\|u\|_{2, \alpha}
$$

provided that $2 \alpha / s \leq 6 /[3-2(\alpha-s)]$, or that $6(\alpha-s) \leq 4 \alpha(\alpha-s)$, i.e. $6 \leq 4 \alpha$, which is true e.g. if $\alpha \geq 2$. For the right-hand side of (2.32) we can replace $\|u\|_{2, \alpha}$ by

$$
\|u\|_{2, \alpha, s} \equiv\left(\left\|D^{s} u\right\|_{2}^{2}+\left\|D^{s+1} u\right\|_{2}^{2}+\cdots+\left\|D^{\alpha} u\right\|_{2}^{2}\right)^{1 / 2}
$$

since (2.32) can be obtained from the inequality $\|v\|_{2 \alpha / s} \leq C_{s}\|v\|_{2, \alpha-s}$ by replacing $v$ by $D^{s} u$. Combining (2.30)-(2.33) we obtain (2.28), since $i+j+h+\cdots+k=\beta \leq \alpha$, thus proving the lemma.

We now suppose we have smooth functions $g_{k}:\left[-U_{k}, U_{k}\right] \rightarrow \mathbb{R}$ whose derivatives are all bounded on $\left[-U_{k}, U_{k}\right]$ and that we have smooth functions $u_{k}: \Omega \rightarrow$ $\left[-U_{k}, U_{i}\right], k=1,2, \ldots, N$. The next lemma estimates derivatives of the function $g_{1}\left(u_{1}\right) g_{2}\left(u_{2}\right) \cdots g_{N}\left(u_{N}\right)$.

Lemma 2.3. Let $g_{k}, u_{k}$ be as above, $1 \leq k \leq N$. Then for each integer $\alpha \geq 1$ there exists a constant $G_{\alpha, N}$ such that

$$
\left\|D^{\alpha}\left[\prod_{k=1}^{N} g_{k}\left(u_{k}\right)\right]\right\|_{2} \leq G_{\alpha, N}\left(\sum_{k=1}^{N}\left(\left\|D u_{k}\right\|_{2}^{2}+\cdots+\left\|D^{\alpha} u_{k}\right\|_{2}^{2}\right)\right)^{\alpha / 2} .
$$

Proof. We use induction on $N$. Lemma 2.2 handles the case $N=1$. We now assume the result for $N$ and attempt to infer from this the result for $N+1$. Letting $G_{N}(u)=g_{1}\left(u_{1}\right) \cdots g_{N}\left(u_{N}\right)$, we have from the Leibniz rule that

$$
\begin{aligned}
&\left\|D^{\alpha}\left[\prod_{k=1}^{N+1} g_{k}\left(u_{k}\right)\right]\right\|=\left\|D_{2}^{\alpha}\left(G_{N}(u) g_{N+1}\left(u_{N+1}\right)\right)\right\|_{2} \\
&=\left\|\sum_{k=0}^{\alpha}\left(\begin{array}{l}
\alpha \\
k
\end{array}\right) D_{\alpha-k}\left(G_{N}(u)\right) D_{k}\left(g_{N+1}\left(u_{N+1}\right)\right)\right\|_{2} \\
& \leq\left\|\left(D_{\alpha}\left(G_{N}(u)\right)\right) g_{N+1}\left(u_{N+1}\right)\right\|_{2}+\left\|G_{N}(u) D_{\alpha}\left(g_{N+1}\left(u_{N+1}\right)\right)\right\|_{2} \\
&+\sum_{k=1}^{\alpha-1}\left(\begin{array}{l}
\alpha \\
k
\end{array}\right)\left\|D_{\alpha-k} G_{N}(u) D_{k}\left(g_{N+1}\left(u_{N+1}\right)\right)\right\|_{2} \\
& \leq\left\|g_{N+1}\left(u_{N+1}\right)\right\|_{\infty}\left\|D_{\alpha} G_{N}(u)\right\|_{2}+\left\|G_{N}(u)\right\|_{\infty}\left\|D_{\alpha}\left(g_{N+1}\left(u_{N+1}\right)\right)\right\|_{2} \\
&+\sum_{k=1}^{\alpha-1}\left(\begin{array}{l}
\alpha \\
k
\end{array}\right)\left\|D_{\alpha-k} G_{N}(u)\right\|_{4}\left\|D_{k}\left(g_{N+1}\left(u_{N+1}\right)\right)\right\|_{4} .
\end{aligned}
$$

But by the Sobolev inequalities and previous remarks there exists a constant $C_{\alpha, k}$ such that

$$
\begin{aligned}
& \left\|D_{\alpha-\beta} G_{N}(u)\right\|_{4}\left\|D_{k}\left(g_{N+1}\left(u_{N+1}\right)\right)\right\|_{4} \\
& \quad \leq C_{\alpha, k}\left\|G_{N}(u)\right\|_{2, \alpha+1-k, \alpha-k}\left\|g_{N+1}\left(u_{N+1}\right)\right\|_{2, k+1, k}
\end{aligned}
$$


where $\|\cdot\|_{2, \beta, s}$ is defined as in (2.33). Using the boundedness of $g_{N+1}\left(u_{N+1}\right)$ and $G_{N}(u)$ and combining $(2.35),(2.36)$, the inductive hypothesis on $G_{N}(u)$, and the results of Lemma 2.2, we obtain (2.34) and thus prove Lemma 2.3.

We now are in a position to bootstrap (2.26) and (2.27) into higher-order estimates on $T$ and the $Y_{i}$. We begin with an inductive hypothesis on $T$ and the $Y_{i}$; we suppose that there exists for the integer $\alpha \geq 0$ a constant $C_{\alpha}^{u}$ such that, for $A \equiv-\Delta$,

$$
\left\|A^{\alpha / 2} u(\cdot, t)\right\|_{2}^{2}+d_{u} \int_{0}^{t}\left\|A^{(\alpha+1) / 2} u\right\|_{2}^{2} d s \leq C_{\alpha}^{u},
$$

where $u=T$ or $Y_{i}$ and $d_{u}=1$ or $d_{i}$, respectively. Multiplying both sides of (1.4a) by $A^{\alpha+1} T$, integrating over $\Omega$, and using the self-adjointness of the (fractional powers of) $A$, we have that

$$
\begin{aligned}
\frac{d}{d t} \frac{1}{2}\left\|A^{(\alpha+1) / 2} T\right\|_{2}^{2}= & -\left\|A^{(\alpha+2) / 2} T\right\|_{2}^{2}-\left(A^{\alpha / 2}(v \cdot \nabla T), A^{(\alpha+2) / 2} T\right) \\
& +Q\left(A^{\alpha / 2} \omega, A^{(\alpha+2) / 2} T\right) \\
\leq & -\left\|A^{(\alpha+2) / 2} T\right\|_{2}^{2}+\varepsilon\left\|A^{\alpha / 2}(v \cdot \nabla T)\right\|_{2}^{2} \\
& +\frac{1}{\varepsilon}\left\|A^{(\alpha+2) / 2} T\right\|_{2}^{2}+Q \delta\left\|A^{\alpha / 2} \omega\right\|_{2}^{2} \\
& +\frac{Q}{\delta}\left\|A^{(\alpha+2) / 2} T\right\|_{2}^{2} .
\end{aligned}
$$

Setting $\varepsilon=\frac{1}{4}$ and $\delta=4 Q$ in (2.38), we obtain that

$$
\begin{aligned}
& \frac{d}{d t} \frac{1}{2}\left\|A^{(\alpha+1) / 2} T\right\|_{2}^{2}+\frac{1}{2}\left\|A^{(\alpha+2) / 2} T\right\|_{2}^{2} \\
& \quad \leq 4\left\|A^{\alpha / 2}(v \cdot \nabla T)\right\|_{2}^{2}+4 Q^{2}\left\|A^{\alpha / 2} \omega\right\|_{2}^{2} .
\end{aligned}
$$

To treat the terms on the right-hand side (2.39), we first note that we can basically interchange $D^{\beta} u$ and $A^{\beta / 2} u$ for integers $\beta \geq 1$ in our discussions above and in estimates calculated from use of the Leibniz rule, due to the equivalence of the norms $\left\|A^{\beta / 2}(\cdot)\right\|_{2}$ and $\left\|D^{\beta}(\cdot)\right\|_{2}$ on either $H^{\beta}(\Omega)$, or $H^{\beta}(\Omega)$ intersected with the subspace perpendicular to the constant functions, depending on whether boundary conditions (1.6) or (1.5) are imposed. With this in mind we see from the Leibniz rule that there is a constant $C_{\alpha}^{v}$ such that

$$
4\left\|A^{\alpha / 2}(v \cdot \nabla T)\right\|_{2}^{2} \leq C_{\alpha}^{v}\|T\|_{2,(\alpha+1) / 2,1}^{2}
$$

where $C_{\alpha}^{v}$ depends primarily on $\left\|D^{\beta} v\right\|_{\infty}, 0 \leq \beta \leq \alpha$; note our assumptions on $v$ guarantee that the latter norms are bounded. Integrating both sides of (2.40) over $[0, t]$ and using (2.37), we have that

$$
\begin{aligned}
4 \int_{0}^{t}\left\|A^{\alpha / 2}(v \cdot \nabla T)\right\|_{2}^{2} d s & \leq C_{\alpha}^{v}\left(C_{1}^{T}+C_{2}^{T}+\cdots+C_{\alpha}^{T}\right) \\
& \equiv C_{\alpha}^{v, T}
\end{aligned}
$$

For the second term on the right-hand side of (2.39) we use Lemma 2.3 with $M+1=$ $N, u_{k}=Y_{k}, k=1, \ldots, M, u_{M+1}=T$, and with the obvious choices for the $g_{k}$; we 
then have from that lemma and (2.37) that

$$
\begin{aligned}
& 4 Q^{2}\left\|A^{\alpha / 2} \omega\right\|_{2}^{2} \leq G_{\alpha, M+1}\left[\sum_{k=1}^{M}\left(\sum_{\beta=1}^{\alpha}\left\|A^{\beta / 2} Y_{k}\right\|_{2}^{2}\right)^{\alpha}\right]\left(\sum_{\beta=1}^{\alpha}\left\|A^{\beta / 2} T\right\|_{2}^{2}\right)^{\alpha} \\
& \leq G_{\alpha, M+1}\left[\sum_{k=1}^{M}\left(\sum_{\beta=1}^{\alpha} C_{\beta}^{Y_{k}}\right)^{\alpha}\right]\left(\sum_{\beta=1}^{\alpha} C_{\beta}^{T}\right)^{\alpha-1} \sum_{\beta=1}^{\alpha}\left\|A^{\beta / 2} T\right\|_{2}^{2} \\
& \equiv G_{\alpha, M+1}^{Y, T} \sum_{\beta=1}^{\alpha}\left\|A^{\beta / 2} T\right\|_{2}^{2} .
\end{aligned}
$$

Integrating (2.42) over $[0, t]$ and again using $(2.37)$, we have that

$$
\begin{aligned}
4 Q^{2} \int_{0}^{t}\left\|A^{\alpha / 2} \omega\right\|_{2}^{2} d s & \leq G_{\alpha, M+1}^{Y, T}\left(C_{0}^{T}+C_{1}^{T}+\cdots+C_{\alpha-1}^{T}\right) \\
& \equiv C_{\alpha}^{Y, T} .
\end{aligned}
$$

Integrating (2.39) over $[0, t]$ and using (2.41) and (2.43), we thus obtain that

$$
\begin{aligned}
\left\|A^{(\alpha+1) / 2} T\right\|_{2}^{2}+\int_{0}^{t}\left\|A^{(\alpha+2) / 2} T\right\|_{2}^{2} d s & \leq\left\|A^{(\alpha+1) / 2} T_{0}\right\|_{2}^{2}+2\left(C_{\alpha}^{v, T}+C_{\alpha}^{Y, T}\right) \\
& \equiv C_{\alpha+1}^{T} .
\end{aligned}
$$

Hence by induction (2.37) holds for $u=T$ for as high an $\alpha$ as the smoothness of $v$ permits; a similar argument reaches the same conclusion regarding (2.37) for $u=Y_{i}, 1 \leq i \leq M$.

Since the estimate (2.37) is now established with $u=T$ or $Y_{i}$ and $d_{u}=1$ or $d_{i}$ for any $\alpha \geq 0$, we then have that (2.7) and (2.8) follow from the Sobolev inequalities. This establishes Proposition 2.1, and this result will turn out to be critical in establishing our next lemma, which shows that asymptotically our solutions $T$ and $Y_{i}$ behave like their spatial averages. The idea of using such a result in studying various reaction-diffusion equations modeling combustion phenomena first appeared in a paper by Fitzgibbon and Martin (see [10, Lemma 2.5]), and we have subsequently applied suitable modifications of their lemma in further applications ([4], [5]). In proving Proposition (2.1) we have obtained the estimates necessary to allow us to prove such a lemma for (1.4) in the case of nonzero $v$. For ease of notation in stating the result, we let $\bar{T}$ and $\bar{Y}_{i}$ denote the spatial averages of $T$ and $Y_{i}$, i.e. (1.11) with $h$ replaced by $T$ and $Y_{i}$, respectively.

Lemma 2.4. Let $T$ and $Y_{i}, 1 \leq i \leq M$, be as in Theorem 1.2, and let $\bar{T}$ and $\bar{Y}_{i}$ be as above. Then we have that

$$
\lim _{t \rightarrow \infty}\left\|Y_{i}(\cdot, t)-\bar{Y}_{i}(t)\right\|_{\infty}=0
$$

and if conditions (1.5) are assumed, we also have that

$$
\lim _{t \rightarrow 0}\|T(\cdot, t)-\bar{T}(t)\|_{\infty}=0 .
$$

Proof. Let $u=Y_{i}, 1 \leq i \leq M$, or $T$ under the conditions (1.5). Then from (2.12) and (2.21) we have that

$$
\int_{0}^{\infty} \int_{\Omega}|\nabla u|^{2} d x d s<\infty
$$


Applying $\nabla$ to both sides of the appropriate equation in (1.4), we see that

$$
\begin{array}{r}
\left\|\nabla u_{t}\right\|_{\infty} \leq d_{u}\|\nabla(\Delta u)\|_{\infty}+\|\nabla(v \cdot \nabla u)\|_{\infty}+Q_{u}\|\nabla \omega\|_{\infty} \\
\leq C_{u}\left(\left\|A^{3 / 2} u\right\|_{\infty}+\left\|A^{1 / 2}(v \cdot \nabla u)\right\|_{\infty}+\left\|A^{1 / 2} \omega\right\|_{\infty}\right)
\end{array}
$$

where the constant $C_{u}$ comes from the various equivalences of norms discussed earlier; here $Q_{u}=Q$ or $Q_{u}=\alpha_{i}$, according to whether $u=T$ or $Y_{i}$. But by the calculations in Lemmas 2.2 and 2.3 the terms $\left\|A^{1 / 2}(v \cdot \nabla u)\right\|_{\infty}$ and $\left\|A^{1 / 2} \omega\right\|_{\infty}$ can be estimated in terms of $\left\|A^{1 / 2} T\right\|_{\infty}$ and $\left\|A^{1 / 2} Y_{i}\right\|_{\infty}$, both of which are bounded by virtue of (2.7) and (2.8). Thus from (2.48) we see that $\left\|\nabla u_{t}(\cdot, t)\right\|_{\infty}$ is uniformly bounded for all $t \geq 0$. From this uniform bound, from (2.47), and from Hölder's inequality we thus obtain that

$$
\lim _{t \rightarrow \infty}\||\nabla u(\cdot, t)|\|_{p}=0
$$

for all $p \geq 1$.

Let $\gamma_{1}$ be the first positive eigenvalue of the operator $-d_{u} \Delta$ under the conditions (1.5); then by eigenfunction expansion we see that

$$
\gamma_{1}\|u(\cdot, t)-\bar{u}(t)\|_{2} \leq\||\nabla u(\cdot, t)|\|_{2}^{2}
$$

and hence

$$
\lim _{t \rightarrow \infty}\|u(\cdot, t)-\bar{u}(t)\|_{2}=0 .
$$

Since $\|u(\cdot, t)\|_{\infty}$ is uniformly bounded by virtue of (2.7) and (2.8), we then have from (2.51) and Hölder's inequality that

$$
\lim _{t \rightarrow \infty}\|u(\cdot, t)-\bar{u}(t)\|_{p}=0
$$

for all $p \geq 1$. Selecting $p>3$, we have, again from the Sobolev inequalities, that there exists a constant $K_{p}$ such that

$$
\|u(\cdot, t)-\bar{u}(t)\|_{\infty} \leq K_{p}\left[\|u(\cdot, t)-\bar{u}(t)\|_{p}+\||\nabla u(\cdot, t)|\|_{p}\right],
$$

where we note that $\nabla \bar{u}(t)=0$. Thus from (2.49), (2.52), and (2.53) we obtain that

$$
\lim _{t \rightarrow \infty}\|u(\cdot, t)-\bar{u}(t)\|_{\infty}=0 \text {, }
$$

which proves the lemma.

This last lemma is one of the main tools we use in the next section to exploit the identities (2.3) and (2.5) in proving Theorem 1.2. The proof we employed here for Lemma 2.4 is only a slight modification of the proof of [10, Lemma 2.5]. Again, one of the main goals in this section was actually to obtain Proposition 2.1 so as to make the bound (2.48) possible.

\section{Proof of Theorem 1.2}

We first show that, under the conditions of Theorem 1.1, there exist constants $\alpha>0$ and $t_{1} \geq 0$ such that $T(x, t) \geq \alpha$ for all $x$ in $\Omega$ and all $t \geq t_{1}$. The presence of nonzero $v$ in (1.4) makes this somewhat less trivial to prove than was the case in [4]. We begin with the case of boundary conditions (1.5).

Under these conditions, consider the (time-dependent) operator $A_{v}=\Delta-v \cdot \nabla$, and let $u$ solve the initial-value problem

$$
\begin{gathered}
u_{t}=A_{v} u, \\
u(x, 0)=u_{0},
\end{gathered}
$$


where $u_{0} \in C(\bar{\Omega})$. Invoking Lemma 2.4 applied to (1.4a) in the case $Q=0$, we see that

$$
\lim _{t \rightarrow \infty}\|u(\cdot, t)-\bar{u}(t)\|_{\infty}=0
$$

where, as before, $\bar{u}$ is the spatial average of $u$. But, by integrating both sides of (3.1a) over $\Omega$, we see that

$$
\bar{u}(t)=\left(u_{0}\right)_{A V}
$$

as a special case of $(2.5)$. Thus let $V(t, s)$ be the fundamental solution generated by $A_{v}$ so that $u(t)=V(t, 0) u_{0}$; then from (3.2) and (3.3) we see that

$$
\lim _{t \rightarrow \infty} V(t, 0) u_{0}=\left(u_{0}\right)_{A V} .
$$

We note that $T$ satisfies the integral equation

$$
T(t)=V(t, 0) T_{0}+Q \int_{0}^{t} V(t, s) \omega(s) d s
$$

where we have suppressed the dependence on $x$. From the maximum principle $V(t, s)$ is positivity-preserving; since $\omega(s) \geq 0$ we thus have from (3.5) that

$$
T(x, t) \geq\left(V(t, 0) T_{0}\right)(x)
$$

for all $x$ in $\Omega$ and all $t \geq 0$. Applying (3.4) with $T_{0}=u_{0}$, we see from (3.6) that, for $\alpha=\left(T_{0}\right)_{A V}-\varepsilon$ for any $\varepsilon$ with $0<\varepsilon \ll\left(T_{0}\right)_{A V}$, we have that there exists a $t_{1}$ so that $T(x, t) \geq \alpha$ for all $t \geq t_{1}$ as desired; note that $\left(T_{0}\right)_{A V}>0$ since $T_{0}$ is not identically zero.

We now consider (3.1) under the boundary conditions (1.6). Let $\lambda_{1}$ be the first eigenvalue of $-\Delta$; then, multiplying both sides of (3.1a) by $u$ and integrating over $\Omega$, we have that

$$
\begin{aligned}
\frac{d}{d t} \frac{1}{2}\|u\|_{2}^{2}=(\Delta u, u)+((v \cdot \nabla) u, u) \\
=(\Delta u, u)+((\operatorname{div} v) u, u) \\
=(\Delta u, u) \leq-\lambda_{1}\|u\|_{2}^{2} .
\end{aligned}
$$

Thus from (3.7) we see that, for $\beta_{1}=\sqrt{2} \lambda_{1}$,

$$
\left\|V(t, 0) u_{0}\right\|_{2} \leq\left\|u_{0}\right\|_{2} e^{-\beta_{1} t}
$$

for all $t \geq 0$.

But from the Sobolev inequalities we see that there exists a constant $C_{3}$ such that

$$
\|u\|_{\infty} \leq C_{3}\left\|D^{3} u\right\|_{2}^{1 / 2}\|u\|_{2}^{1 / 2} .
$$

But $\left\|D^{3} u\right\|_{2}$ is uniformly bounded by (2.37) in the special case $Q=0$. Hence from (3.8) and (3.9) we see that there is a constant $C_{3}^{u}$ such that

$$
\begin{aligned}
\left\|V(t, 0) u_{0}\right\|_{\infty}^{2} & \leq C_{3}^{u}\left\|u_{0}\right\|_{2} e^{-\beta_{1} t} \\
& \leq|\Omega|^{1 / 2} C_{3}^{u}\left\|u_{0}\right\|_{\infty} e^{-\beta_{1} t} \\
& \equiv K_{3}^{u}\left\|u_{0}\right\|_{\infty} e^{-\beta_{1} t}
\end{aligned}
$$

for all $t \geq 0$. 
Let $w$ be as in (1.10); then $T$ satisfies the integral equation

$$
T(t)=V(t, 0)\left(T_{0}-w\right)+w+Q \int_{0}^{t} V(t, s) \omega(s) d s .
$$

Again, $V(t, s)$ is positivity-preserving; thus from (3.11) we see that

$$
T(x, t) \geq\left(V(t, 0)\left(T_{0}-w\right)\right)(x)+w(x)
$$

for all $x \in \Omega$ and all $t \geq 0$. Since $\partial \Omega$ is compact there exists an $x_{0} \in \partial \Omega$ such that $g(x) \geq g\left(x_{0}\right)>0$ for all $x \in \partial \Omega$. By the maximum principle we thus have that $w(x) \geq g\left(x_{0}\right)$ for all $x \in \Omega$. Substituting $T_{0}-w$ for $u_{0}$ in (3.10), we see that the first term on the right-hand side of (3.12) converges to zero uniformly as $t \rightarrow \infty$; thus again we have $T(x, t)$ eventually uniformly bounded below by a positive constant $\alpha$. We summarize the above observations under both sets of boundary conditions as a formal result:

Proposition 3.1. Under the conditions of Theorem 1.1 we have that there exist constants $\alpha>0$ and $t_{1} \geq 0$ such that $T(x, t) \geq \alpha$ for all $x$ in $\Omega$ and all $t \geq t_{1}$.

The rest of the proof of Theorem 1.2 closely resembles the proof in the case $v=0$ found in [4] once we note that the same basic comparison principles hold for (1.4). Let $\left(Z_{i 0}\right)_{A V}$ be as in the discussion preceding Theorem 1.2; then $\left(Z_{i 0}\right)_{A V} \geq 0$. Taking into account the possible "critical" cases $\left(Z_{i 0}\right)_{A V}=0$-by relabeling, if necessary - we can assume that $\left(Z_{i 0}\right)_{A V}=0, i=2, \ldots, M_{0}$, for some $M_{0}$ with $2 \leq M_{0} \leq M$, and that $\left(Z_{i 0}\right)_{A V}>0, i=M_{0}+1, \ldots, M$. Combining (2.3) and (2.45), we have that

$$
\lim _{t \rightarrow \infty}\left\|Y_{i}(\cdot, t)-\left(\alpha_{i} / \alpha_{1}\right) Y_{1}(\cdot, t)\right\|_{\infty}=0, \quad 2 \leq i \leq M_{0} .
$$

Thus given $\varepsilon>0$ there exists a constant $t_{i} \geq 0$ such that

$$
Y_{i}(x, t) \geq\left(\alpha_{i} / \alpha_{1}\right)\left(Y_{1}(x, t)-\varepsilon\right), \quad 2 \leq i \leq M_{0},
$$

for all $x \in \Omega$ and all $t \geq t_{i}$. For the other $Y_{i}$ we first note that from (2.3) we have that

(3.15) $\bar{Y}_{i}(x, t)=\left(\alpha_{i} / \alpha_{1}\right) \bar{Y}_{1}(x, t)+\left(z_{i 0}\right)_{A V} \geq\left(z_{i 0}\right)_{A V}>0, \quad M_{0}+1 \leq i \leq M$,

for all $x \in \Omega$ and all $t \geq 0$. Thus from (3.15) and (2.45) we see that there exist constants $b_{i}>0$ and $t_{i}, M_{0}+1 \leq i \leq M$, such that

$$
Y_{i}(x, t) \geq b_{i}
$$

for all $x$ in $\Omega$ and all $t \geq t_{i}$. Let $t_{1}$ and $\alpha$ be as in Proposition 3.1, and set $t_{0}=\max \left\{t_{1}, t_{2}, \ldots, t_{M}\right\}, d=\min \left\{\alpha_{2} / \alpha_{1}, \ldots, \alpha_{M} / \alpha_{1}\right\}, \nu_{0}=\nu_{2}+\cdots+\nu_{M_{0}}$, and $c=\alpha_{1}\left[\prod_{i=M_{0}+1}^{M} b_{i}^{\nu_{i}}\right] d^{\nu_{0}} f(\alpha)$. Then since the maximum principle still holds for (1.4b), so do standard comparison principles, and they now assert that if $u=u(t)$ solves the ODE initial-value problem

$$
\begin{gathered}
\dot{u}=-c u^{\nu_{1}}(u-\varepsilon)^{\nu_{0}}, \\
u(0)=\left\|Y_{10}\right\|_{\infty},
\end{gathered}
$$

then for $t \geq t_{0} u$ is a supersolution of (1.4b) with $i=1$ (note $A_{v} u=0$ ); i.e. for all $x$ in $\Omega$ and all $t \geq t_{0}$ we have that

$$
0 \leq Y_{1}(x, t) \leq u\left(t-t_{0}\right) .
$$


Setting $u_{\varepsilon}(t)=u(t)-\varepsilon$, we can rewrite (3.17a) as

$$
\dot{u}_{\varepsilon}=-c\left(u_{\varepsilon}+\varepsilon\right)^{\nu_{1}}\left(u_{\varepsilon}\right)^{\nu_{0}} .
$$

Then $u_{\varepsilon}(t) \leq \bar{u}_{\varepsilon}(t)$ where $\bar{u}_{\varepsilon}$ solves the ODE initial-value problem

$$
\begin{aligned}
\dot{\bar{u}}_{\varepsilon} & =-c\left(\bar{u}_{\varepsilon}\right)^{\nu}, \\
\bar{u}_{\varepsilon}(0) & =\left\|Y_{10}\right\|_{\infty}-\varepsilon
\end{aligned}
$$

where $\nu=\nu_{1}+\nu_{0}$. Since $\nu_{i} \geq 1,1 \leq i \leq M$, we have that $\nu>1$, in which case $\bar{u}_{\varepsilon}(t)=(a t+b)^{-\gamma}$ with $\gamma=1 /(\nu-1), a=c /(\nu-1)$, and $b=\left(\left\|Y_{10}\right\|_{\infty}-\varepsilon\right)^{1-\nu}$. Since $u(t)=u_{\varepsilon}(t)+\varepsilon \leq \bar{u}_{\varepsilon}(t)+\varepsilon$, we have from (3.18) that for all $x$ in $\Omega$ and all $t \geq t_{0}$

$$
0 \leq Y_{1}(x, t) \leq\left(a\left(t-t_{0}\right)+b\right)^{-\nu}+\varepsilon .
$$

Since $\varepsilon$ is arbitrary we can conclude from (3.21) that

$$
\lim _{t \rightarrow \infty} Y_{1}(x, t)=0
$$

uniformly in $x$. Thus $Y_{i}(x, t) \rightarrow 0$ uniformly, $2 \leq i \leq M_{0}$, by (3.13), while (1.12) and (1.13) follow from (2.3), (2.5), (2.45), (2.46), (3.22), and the bounded convergence theorem. For the boundary conditions (1.6a) we use (3.11); we already have that $V(t, 0)\left(T_{0}-w\right) \rightarrow 0$ uniformly as $t \rightarrow \infty$, so it remains to show that the same holds for the integral term on the right-hand side of (3.11). But from (3.10) we have that

$$
0 \leq \int_{0}^{t} V(t, s) \omega(s) d s \leq\left(K_{3}^{T}\right) \int_{0}^{t} e^{-\beta_{2}(t-s)}\|\omega(s)\|_{\infty}^{1 / 2} d s
$$

where $\beta_{2}=\beta_{1} / 2$. Note that $\|\omega(s)\|_{\infty}^{1 / 2} \rightarrow 0$ as $t \rightarrow \infty$ since $Y_{1}^{\nu_{1}} \rightarrow 0$ and $f(T)$ and the other $Y_{i}^{\nu_{i}}$ are uniformly bounded. Thus the right-hand side of (3.23) converges to zero uniformly as $t \rightarrow \infty$ by [5, Lemma 1.3]; the latter result is a simple consequence of the dominated convergence theorem.

Combining the above remarks, we see that all time-dependent terms in (3.11) converge to zero uniformly as $t \rightarrow \infty$, from which we obtain (1.14); this completes the proof of the theorem.

\section{Remarks}

We have mentioned earlier that the steady-state convergence results obtained in [3], [5], [10] for multiple-step chemical reactions with $v=0$ can probably be reestablished for nontrivial $v$ using the techniques developed here. This can be accomplished on a case-by-case basis by proving appropriate versions of (2.3), (2.5), (2.9), (2.12), and (2.21) adapted to fit the particular reaction-convection-diffusion system modeling each reaction. Thenceforth the establishment of the key inequality (2.37) would be a straightforward generalization of the calculations done in Section 2 above.

Setting $\alpha=0$ in (2.7), we obtain a uniform bound on $T$. Obtaining such a bound in a general class of reaction-diffusion equations that includes (1.4) with $v=0$ is a problem that has attracted a substantial amount of activity beginning with [1]; also see [10] for further references and remarks. Here we have had to create a more general iterative process that allows us to handle nontrivial convection terms.

We note again that the main thrust of Theorem 1.2 is that the asymptotic behavior of the system (1.4) is unchanged when convection terms are added, no matter what the convection terms provide in terms of inhibitive or enhancing effects 
on mixing over intermediate time scales. It would be interesting to investigate, even by an informal asymptotic analysis, what significant intermediate effects are in fact possible due to the convection terms.

\section{REFERENCES}

1. N. Alikakos, An application of the invariance principle to reaction-diffusion equations, J. Differential Equations 33 (1979), 201-225. MR 80m:35011

2. J.D. Avrin, Qualitative theory for a model of laminar flames with arbitrary nonnegative initial data, J. Differential Equations 84 (1990), 290-308. MR 91h:35316

3. __ Decay and boundedness results for a model of laminar flames with complex chemistry, Proc. Amer. Math. Soc. 110 (1990), 989-995. MR 91c:80017

4. _ Asymptotic behavior of one-step combustion models with multiple reactants on bounded domains, SIAM J. Math. Anal. 24 (1993), 290-298. MR 94k:80008

5. __ Asymptotic behavior of some reaction-diffusion systems modelling complex combustion on bounded domains, Proc. Roy. Soc. Edinburgh 123A (1993), 1151-1163. MR 95a:35071

6. L Large-eigenvalue global existence and regularity results for the Navier-Stokes equations, J. Differential Equations 127 (1996), 365-390. MR 97b:35138

7. H. Berestycki, B. Nicolaenko and B. Scheurer, Traveling wave solutions to combustion models and their singular limits, SIAM J. Math. Anal. 16 (1985), 1207-1242. MR 87h:35326

8. J. D. Buckmaster and G. S. S. Ludford, Lectures on Mathematical Combustion, CBMS-NSF Regional Conference Series in Applied Mathematics 43, SIAM, Philadelphia, 1983. MR 86j: 80010

9. P. V. Danckwerts, Gas-Liquid Reactions, McGraw-Hill, New York, 1970.

10. W. E. Fitzgibbon and C. B. Martin, Semilinear parabolic systems modelling spatially inhomogeneous exothermic reactions, J. Math. Anal. Appl. 178 (1993), 165-175. MR 94h:35118

11. The longtime behavior of solutions to a quasilinear combustion model, Nonlinear Anal. 19 (1992), 947-961. MR 93m:80012

12. H. Fujita and T. Kato, On the Navier-Stokes initial value problem I, Arch. Rational Mech. Anal. 16 (1964), 269-315. MR 29:3774

13. I. M. Gelfand, Some problems in the theory of quasilinear equations, Amer. Math. Soc. Translations (2) 29 (1963), 295-381. MR 27:3921

14. Y. Giga and T. Miyakawa, Solutions in $L_{r}$ of the Navier-Stokes initial value problem, Arch. Rational Mech. Anal. 89 (1985), 267-281. MR 86m:35138

15. C. Guillopé, Comportement à l'infini des solutions des équations de Navier-Stokes et propriété des ensembles fonctionnels invariants (ou attracteurs), Ann. Inst. Fourier (Grenoble) 32 (1982), 1-37. MR 84a:35241

16. I. S. Gradshteyn and I. M. Ryzhik, Tables of Integrals, Series, and Products (A. Jeffrey, ed.), Academic Press, New York, 1980. MR 81g:33001

17. D. Henry, Geometric Theory of Semilinear Parabolic Equations, Lecture Notes in Mathematics 840 (Berlin: Springer, 1981). MR 83j:35084

18. O. A. Ladyzhenskaya, The Mathematical Theory of Viscous Imcompressible Flow, 2nd ed. (English translation), Gordon and Breach, New York, 1969. MR 40:7610

19. B. Larrouturou, The equations of one-dimensional unsteady flame propagation: existence and uniqueness, SIAM J. Math. Anal. 19 (1988), 32-59. MR 89i:80002

20. M. Marion, Attractors for reaction-diffusion equations: existence and estimate of their dimension, Appl. Anal. 25 (1987), 101-147. MR 88m:35082

21. G. Raugel and G. R. Sell, Navier-Stokes equations on thin 3D domains I: global attractors and global regularity of solutions, AHPCRC Preprint 90-04, J. Amer. Math. Soc. 6 (1993), 503-568. MR 93j:35134

22. J. M. Roquejoffre, Thesis, INRIA Sophis Antipolis, June 1988.

23. D. Sattinger, A nonlinear parabolic system in the theory of combustion, Q. Appl. Math. 33 (1975/76), 47-61. MR 57:3576

24. J. Smoller, Shock Waves and Reaction-Diffusion Equations, Springer, New York, 1983. MR 84d:35002

25. R. Témam, Navier-Stokes Equations, North-Holland, Amsterdam, 1977. MR 58:29439

26. __ Navier-Stokes Equations and Nonlinear Functional Analysis, CBMS-NSF Regional Conference Series in Applied Mathematics 41, SIAM, Philadelphia, 1983. MR 86f:35152 
27. D. Terman, Connection problems arising from nonlinear diffusion equations, Proceedings of the Microconference on Nonlinear Diffusion, J. Serrin, L. Peletier, W.-M. Ni, eds., SpringerVerlag, Berlin-Heidelberg-New York, 1988. MR 90c:35125

28. Anal. 19 (1988), 1057-1080. MR 89j:35071

29. D. H. Wagner, Premixed laminar flames are traveling waves, Reacting Flows: Combustion and Chemical Reactors, G.S.S. Ludford, ed., Lectures in Applied Mathematics 24 Amer. Math. Soc., Providence, RI, 1986. MR 87e:80018

30. F. Williams, Combustion Theory, 2nd ed., Addison-Wesley, Reading, MA, 1985.

Department of Mathematics, University of North Carolina at Charlotte, CharLOTTE, NorTh CARolina 28223

E-mail address: fma00jda@unccvm.uncc.edu 